\title{
Acúmulo e exportação de macronutrientes em melancia sem sementes
}

\author{
Leilson C. Grangeiro'; Arthur B. Cecílio Filho ${ }^{2}$
}

${ }^{1}$ ESAM, Depto. Fitotecnia, C. Postal 137, 59625-900 Mossoró-RN; E-mail: leilson@esam.br; ${ }^{2}$ UNESP-FCAV, Depto. Prod. Vegetal, Via de acesso Prof. Paulo Donato Castellane, s/n, 14884-900 Jaboticabal-SP; Bolsista Pesquisador - CNPq; E-mail: rutra@fcav.unesp.br

\section{RESUMO}

Com o objetivo de determinar o acúmulo e a exportação de macronutrientes em melancia sem sementes, híbrido Nova, conduziu-se um experimento em Itápolis (SP), de outubro a dezembro de 2001, em um ARGISSOLO VERMELHO-AMARELO Distrófico. As amostragens de plantas foram realizadas aos $15 ; 30 ; 45 ; 60$ e 70 dias após transplantio (DAT), sendo quantificados o acúmulo de massa seca e de macronutrientes na folha, caule + ramos e frutos. $\mathrm{O}$ acúmulo de peso seco foi lento até $30 \mathrm{DAT}$, intensificando-se a partir deste, alcançando no final do ciclo o total de $545,1 \mathrm{~g}$ planta $^{-1}$, onde as folhas participaram com $30 \%$, caule + ramos com $31 \%$ e os frutos com $39 \%$. Até 30 DAT, o acúmulo de nutrientes também foi pequeno, sendo que com a frutificação, houve um forte incremento na quantidade de nutrientes acumulados, com maior demanda no período de 45 a 60 DAT. A ordem decrescente dos macronutrientes acumulados pela cultura foi: $\mathrm{K}>\mathrm{N}>\mathrm{Ca}>\mathrm{P}>\mathrm{Mg}>\mathrm{S}$. Os frutos exportaram 20,3 $\mathrm{kg} \mathrm{ha}^{-1}$ de N; 4,0 $\mathrm{kg} \mathrm{ha}^{-1}$ de P; 26,6 $\mathrm{kg} \mathrm{ha}^{-1}$ de K; 1,4 $\mathrm{kg} \mathrm{ha}^{-1}$ de $\mathrm{Ca} ; 1,6 \mathrm{~kg} \mathrm{ha}^{-1}$ de $\mathrm{Mg}$ e $1,4 \mathrm{~kg} \mathrm{ha}^{-1}$ de S.

Palavras-chave: Citrullus lanatus, nutrição de plantas, crescimento.

\begin{abstract}
Accumulation and exportation of macronutrients in seedless watermelon

The accumulation and exportation of nutrients of the seedless watermelon hybrid Nova was determined under field conditions. The experiment was carried out in Typic Paleudult soil, in the Itápolis region, São Paulo State, Brazil. Sampling of the plants was done at $15 ; 30 ; 45 ; 60$ and 70 days after transplanting date (DAT) and the accumulation of dry mass and macronutrients was quantified in leaves, stem + branches and fruits. The dry mass accumulation was slow until 30 DAT, intensifying with the beginning of the fruit development. At the end of the cycle, the average contribution of leaves, stems + branches and fruits, was respectively of 30; 31 and $39 \%$. The accumulation of nutrients followed the curve of dry mass accumulation. The larger demand for macronutrients occurred from 45 to 60 DAT. The order of nutrients accumulation for the culture was: $\mathrm{K}>\mathrm{N}>\mathrm{Ca}>\mathrm{P}>\mathrm{Mg}>\mathrm{S}$. The fruits exported $20.3 \mathrm{~kg} \mathrm{ha}^{-1}$ of $\mathrm{N}, 4.0$ $\mathrm{kg} \mathrm{ha}^{-1}$ of P, $26.6 \mathrm{~kg} \mathrm{ha}^{-1}$ of K, $1.4 \mathrm{~kg} \mathrm{ha}^{-1}$ of Ca, $1.6 \mathrm{~kg} \mathrm{ha}^{-1}$ of Mg and $1.4 \mathrm{~kg} \mathrm{ha}^{-1}$ of S.
\end{abstract}

Keywords: Citrullus lanatus, plant nutrition, growth.

\section{(Recebido para publicação em 24 de novembro de 2003 e aceito em 30 de maio de 2005)}

$\mathrm{N}_{\mathrm{s}}^{\mathrm{s}}$ Brasil, a produção de melancia sem sementes é ainda incipiente, embora algumas pequenas áreas comerciais já tenham sido implantadas. $\mathrm{O}$ menor tamanho do fruto, característica que facilita o transporte e acondicionamento, e a ausência de sementes, explorada comercialmente pelas empresas como novidade de mercado, são os principais aspectos que contribuem para a expansão de seu cultivo. Nos Estados Unidos, até 1991, a melancia sem sementes, ocupava cerca de $5 \%$ do mercado de seu fruto, com estimativa de ter potencial para ocupar de 15 a $50 \%$ (MARR; GAST, 1991).

No Brasil, trabalhos sobre marcha de absorção de nutrientes na cultura da melancia foram publicados por Nascimento et al. (1991), com informações sobre a cultura até o início do estádio de frutificação e, mais recentemente, por Grangeiro e Cecílio Filho (2004), que verificaram para o híbrido Tide um crescimento inicial lento, intensificando-se a partir dos 30 dias após o transplantio
(DAT), atingindo, no final do ciclo, um acúmulo de massa seca de 1800 g planta $^{-1}$, sendo a contribuição da parte vegetativa de $31 \%$ e dos frutos de $69 \%$. $\mathrm{O}$ acúmulo de nutrientes também é pequeno no início do ciclo, não ultrapassando $2 \%$ do total até os 30 DAT. Com a frutificação, há um forte incremento na quantidade de nutrientes acumulados, sendo observadas as maiores demandas para $\mathrm{N}, \mathrm{Ca}$ e $\mathrm{Mg}$ no período de 45 a 60 DAT e para P, K e S de 60 a 75 DAT. O acúmulo de nutrientes pela cultura aos 75 DAT é de $139 ; 13 ; 155 ; 25 ; 17$ e 9 kg ha $^{-1}$, respectivamente, de $\mathrm{N}, \mathrm{P}, \mathrm{K}, \mathrm{Ca}$, $\mathrm{Mg}$ e S. Com relação à exportação de nutrientes pelos frutos, os autores observaram que as quantidades foram altas, e representaram importante componente de perdas de nutrientes do solo, sendo de $106,4 \mathrm{~kg} \mathrm{ha}^{-1}$ de $\mathrm{N}, 11,1 \mathrm{~kg} \mathrm{ha}^{-1}$ de $\mathrm{P}, 118,0 \mathrm{~kg} \mathrm{ha}^{-1}$ de $\mathrm{K}, 4,3 \mathrm{~kg} \mathrm{ha}^{-1}$ de $\mathrm{Ca}, 6,8 \mathrm{~kg} \mathrm{ha}^{-1}$ de $\mathrm{Mg}$ e 5,9 $\mathrm{kg} \mathrm{ha}^{-1} \mathrm{de} \mathrm{S}$.

Zhu et al. (1996) afirmam que a taxa de absorção de nutrientes pela cultura acompanha a taxa de produção de mas- sa seca, atingindo o máximo na época do desenvolvimento dos frutos, quando então começa a diminuir. LopezCantarero et al. (1992) verificaram diferenças entre cultivares de melancia para quantidade e teores de nutrientes na folha, sendo observadas diferenças de aproximadamente $100 \%$ nos teores entre as cultivares.

O presente trabalho teve como objetivo determinar o acúmulo e a exportação de macronutrientes pelo híbrido Nova, de melancia sem sementes.

\section{MATERIAL E MÉTODOS}

O trabalho foi conduzido de outubro a dezembro de 2001, em área comercial, localizada no município de Itapólis (SP).O solo foi classificado como ARGISSOLO VERMELHOAMARELO Distrófico, textura média, correspondente ao "Typic Paleudult soil" na classificação americana de solos (SOIL SURVEY SATFF, 1992). A análise química do solo da área expe- 


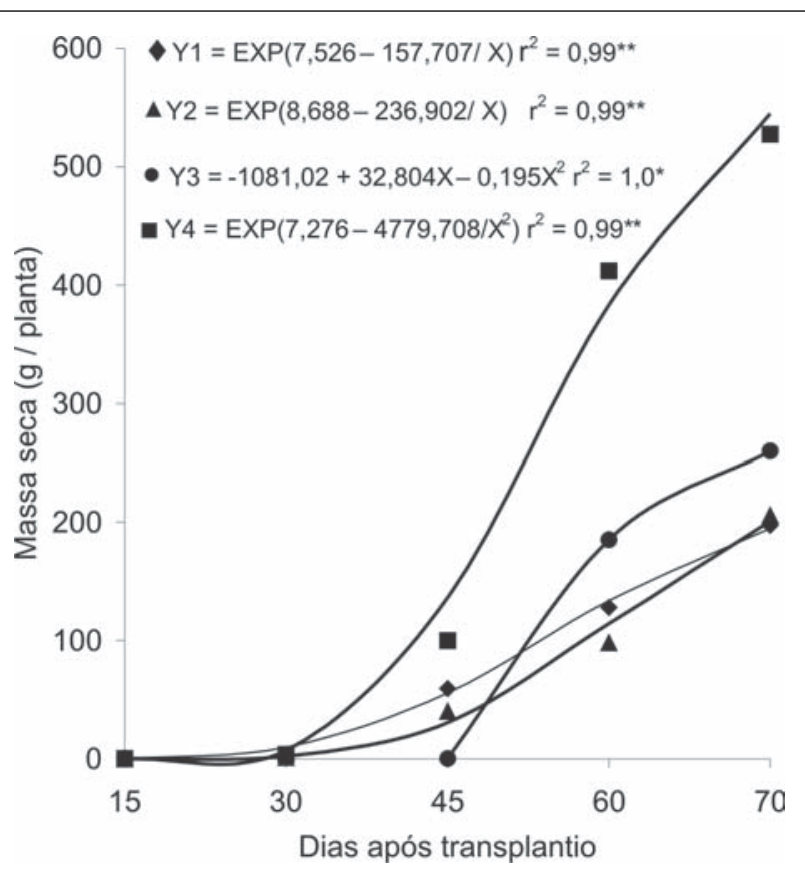

Figura 1. Acúmulo de massa seca nas folhas (Y1), caule + ramos (Y2), frutos (Y3) e planta sem raiz (Y4), de melancia híbrido Nova. Itápolis (SP), UNESP-FCAV, 2001.

rimental revelou: $\mathrm{pH}\left(\mathrm{CaCl}_{2}\right)=4,8 ; \mathrm{P}$ (resina) $=3 \mathrm{mg} \mathrm{dm}^{-3} ; \mathrm{S}=5 \mathrm{mg} \mathrm{dm}^{-3} ;$ M.O. $=$ $11 \mathrm{~g} \mathrm{dm}^{-3} ; \mathrm{K}=1,0 \mathrm{mmol}_{\mathrm{c}}^{-3} ; \mathrm{Ca}=9$ $\mathrm{mmol}_{\mathrm{c}} \mathrm{dm}^{-3} ; \mathrm{Mg}=5 \mathrm{mmol}_{\mathrm{c}} \mathrm{dm}^{-3} ; \mathrm{SB}=15$ mmol $\mathrm{dm}^{-3} ; \mathrm{T}=31 \mathrm{e} \mathrm{V}=48 \%$. As análises foram realizadas em laboratório da UNESP, Jaboticabal, segundo metodologias descritas por Raij et al. (2001).

Após aração e gradagem fez-se a distribuição do calcário dolomítico e a incorporação com grade, 50 dias antes do transplantio em quantidade para elevar a saturação por bases a 70\% (TRANI et al., 1997). Após esse período, procedeu-se a abertura dos sulcos com aproximadamente $30 \mathrm{~cm}$ de profundidade. Baseando-se na recomendação de Trani et al. (1997) e análise química do solo, realizou-se a adubação de plantio com $680 \mathrm{~kg} \mathrm{ha}^{-1}$ do fertilizante N-P-K 4-3010 e adubação de cobertura com $330 \mathrm{~kg}$ ha $^{-1}$ da fórmula 20-00-20, dividida em três parcelas iguais, aplicadas aos 10; 21 e 35 dias após o transplantio (DAT).

A semeadura foi realizada em 03/09/ 2001, em bandejas de poliestireno expandido para 200 mudas, utilizando-se substrato Plantmax ${ }^{\hat{a}}$ e transplantadas aos 30 dias após semeadura, quando apresentavam duas folhas definitivas, no espaçamento 3 x $1 \mathrm{~m}$. Utilizou-se o híbrido Nova, de polpa vermelha e sem sementes e, como polinizador, a cultivar Crimson Sweet, na proporção de uma planta para duas do híbrido Nova.

A partir dos 20 DAT foram feitas adubações foliares semanais, junto com os defensivos, empregando $200 \mathrm{ml}$ por 100 L de solução dos produtos contendo: $0,6 \mathrm{~g} \mathrm{~L}^{-1}$ de $\mathrm{Mg}$; 0,8 $\mathrm{g} \mathrm{L}^{-1}$ de $\mathrm{Ca}$ e 0,05 $\mathrm{g} \mathrm{L}^{-1}$ de B; 0,3 $\mathrm{g} \mathrm{L}^{-1}$ de Zn; 0,2 $\mathrm{g} \mathrm{L}^{-1}$ de Mn e 0,01 $\mathrm{g} \mathrm{L}^{-1}$ de Mo, e volume de $400 \mathrm{~L} \mathrm{ha}^{-1}$. Foram realizadas capinas e penteamento das ramas.

A pluviometria ocorrida no período experimental foi de $306 \mathrm{~mm}$ e não foi realizado irrigação complementar. Esta precipitação foi bem distribuída durante o ciclo cultural.

Os tratamentos foram constituídos pelas épocas de amostragem (15; 30; 45; 60 e 70 dias após o transplantio), sob delineamento de blocos ao acaso, com quatro repetições. Foram coletadas plantas competitivas para quantificação dos teores de macronutrientes e, posteriormente, cálculo do acúmulo e exportação dos mesmos pela melancieira. Nas duas primeiras coletas, em função do pequeno tamanho das plantas, foram amostradas quatro plantas competitivas por repetição e nas avaliações subsequentes duas plantas por repetição.

As plantas coletadas (sem raiz, corte na região do colo) foram fracionadas em folha; caule + ramos e frutos, lavadas em água deionizada e colocadas em estufa com circulação forçada de ar à temperatura de $65^{\circ} \mathrm{C}$. Após a secagem, o material foi moído e digerido para determinação dos teores de N, P, K, Ca, Mg e $\mathrm{S}$ conforme Bataglia et al. (1983). Os dados de massa seca das partes da planta e do acúmulo de nutrientes foram submetidos à análise de regressão, pelo teste F. Se significativo (* teste F, $\mathrm{P}<0,05$ e $* *$ teste $\mathrm{F}, \mathrm{P}<0,01$ ), foram ajustadas equações lineares ou não lineares, com maiores coeficientes de determinação.

\section{RESULTADOS E DISCUSSÃO}

O crescimento da planta de melancia, expresso pelo acúmulo de massa seca, foi lento até 30 dias após transplantio (DAT), intensificando-se a partir deste período. A masa seca total estimada por planta foi de 545,1 g, obtido aos 70 DAT (Figura 1). As folhas participaram com $30 \%$, caule + ramos $31 \%$ e frutos com $39 \%$. No período de 45 a 60 DAT foi verificado o maior acúmulo de massa seca $\left(311,6 \mathrm{~g}\right.$ planta $\left.{ }^{-1}\right)$, correspondendo a aproximadamente $60 \%$ do total (Figura 1).

O acúmulo de massa seca das folhas foi lento até 30 DAT quando, a partir de então, houve um elevado incremento, atingindo, aos 70 DAT, a quantidade estimada de $194,93 \mathrm{~g} \mathrm{planta}^{-1}$. O segmento caule + ramos teve o mesmo comportamento, com massa seca acumulada, no final do ciclo, de 201,03 $\mathrm{g} \mathrm{planta}^{-1}$, superior ao das folhas.

A massa seca acumulada pelos frutos, determinado a partir dos 45 DAT, foi inicialmente pequena, aumentando rapidamente em função do crescimento dos frutos, equivalendo, no final do ciclo a $39 \%$ da massa seca total, com valor estimado de $260 \mathrm{~g} \mathrm{planta}^{-1}$ (Figura 1). O período de maior acúmulo foi de 45 a 60 DAT, apresentando taxa de incremento de $12,3 \mathrm{~g}_{\text {planta }}{ }^{-1} \mathrm{dia}^{-1}$, superior ao verificado nas folhas e caule + ramos, no mesmo período.

As maiores taxas de acúmulo de massa seca nos frutos registradas após a frutificação, sugerem que estes foram os drenos preferenciais da planta. Esta alteração de força de drenos na planta, acontecida com o ingresso da mesma no 
processo reprodutivo, proporciona maior translocação de carboidratos e outros compostos das folhas para os frutos, como decorrência da predominância da fase reprodutiva sobre a fase vegetativa (MARSCHNER, 1995). Fato semelhante foi observado por Souza (1976) em morangueiro, Fayad (1998) em tomateiro e Grangeiro e Cecílio Filho (2002) em melancieira.

A taxa de acúmulo de nutrientes foi pequena nos primeiros 30 DAT, coincidindo com o período de menor acúmulo de massa seca. Os maiores incrementos aconteceram após a frutificação, sendo que a cultura acumulou $80 ; 80 ; 77 ; 84$; 88 e $81 \%$ do total acumulado, respectivamente, de $\mathrm{N}, \mathrm{P}, \mathrm{K}, \mathrm{Ca}, \mathrm{Mg}$ e $\mathrm{S}$, nos últimos 25 dias de ciclo. A ordem dos macronutrientes acumulados foi $\mathrm{K}>\mathrm{N}>\mathrm{Ca}>\mathrm{P}>\mathrm{Mg}>\mathrm{S}$.

Comportamento semelhante foi verificado em melancia por Grangeiro e Cecílio Filho (2002) e em outras espécies da mesma família como melão (LIMA, 2001), pepino (SOLIS et al., 1988) e abobrinha (ARAÚJO et al., 2001). Embora estes trabalhos tenham sido conduzidos em locais, cultivares e sistemas diversos de produção, os mesmos apresentaram similar curva de acúmulo de massa seca e de nutrientes, ou seja, lento acúmulo nos primeiros 30 dias do ciclo e expressivo aumento após o início da frutificação.

O híbrido Nova acumulou 22,7 g planta $^{-1}$ de N, sendo que as folhas foram responsáveis por $42 \%$, caule + ramos $20 \%$ e os frutos com $38 \%$ do acumulado. A maior demanda ocorreu dos 45 aos 60 DAT, coincidindo com a época de maior acúmulo de massa seca na planta (Figura 2A). Em melancia com sementes, Grangeiro e Cecílio Filho (2004) observaram comportamento semelhante, onde o máximo acúmulo de nitrogênio também coincidiu com a fase de maior desenvolvimento da parte vegetativa.

$\mathrm{O}$ acumulo de $\mathrm{N}$ nas folhas foi pequeno nos estádios iniciais, passando de 0,03 g planta $^{-1}$ aos 15 DAT para 9,76 g planta $^{-1}$ aos 70 DAT. No período entre 45 e 60 DAT ocorreu a maior exigência das folhas por este nutriente, com acúmulo estimado de 5,3 $\mathrm{g}_{\text {planta }}{ }^{-1}$, cerca de $54 \%$ do total acumulado pela plan-
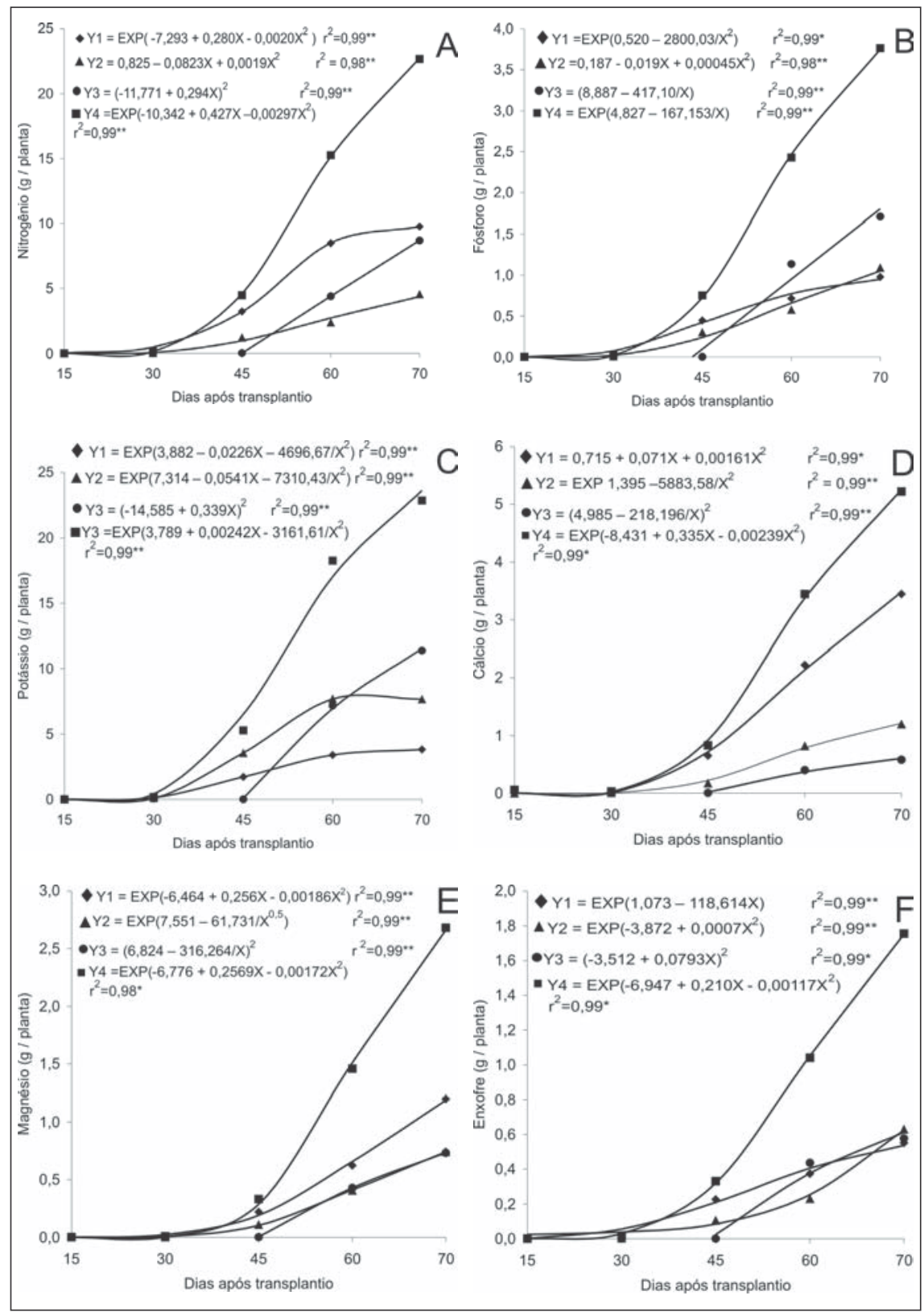

Figura 2. Acúmulo de nitrogênio $(\mathbf{A})$, fósforo $(\mathbf{B})$, potássio $(\mathbf{C})$, cálcio $(\mathbf{D})$, magnésio $(\mathbf{E})$ e enxôfre (F), nas folhas (Y1), ramos (Y2), frutos (Y3) e planta sem raiz (Y4), em melancia, híbrido Nova. Itápolis (SP), UNESP-FCAV, 2001.

ta no referido período. No período seguinte, o acréscimo na quantidade de $\mathrm{N}$ acumulado nas folhas foi menor $(1,3 \mathrm{~g}$ planta $\left.^{-1}\right)$, talvez devido à translocação desse nutriente para os frutos. No caule + ramos, a acumulação de $\mathrm{N}$ aumentou com a idade da planta, onde a quantidade acumulada passou de $0,002 \mathrm{~g}$ planta $^{-1}$ aos 15 DAT para 4,55 g planta $^{-1}$ aos 70 DAT. Nos frutos, apenas em 25 dias de ciclo (45 a 70 DAT) a quantidade acumulada de $\mathrm{N}$ aumentou 870 vezes, a uma taxa média de 347,6 mg N dia-1 (Figura 2A).
O acúmulo de fósforo na planta atingiu valor máximo estimado de $3,75 \mathrm{~g}$ planta $^{-1}$ aos 70 DAT, sendo que as folhas, caule + ramos e frutos contribuíram, respectivamente, com 26, 29 e $45 \%$ (Figura 2B). A maior demanda foi observada no período de 45 a 60 DAT, onde o acúmulo correspondeu a $46 \%$ do total. As folhas e o caule + ramos apresentaram, aproximadamente a mesma quantidade de $\mathrm{P}$ acumulada, com valores no final do ciclo de 0,98 e 1,08 g planta $^{-1}$ respectivamente (Figura 2B). Nos frutos, assim como verificado para 
$\mathrm{N}$, o acúmulo de $\mathrm{P}$ foi linear, sendo estimado aos 45 e 70 DAT acúmulos de 0,03 e 1,75 g planta $^{-1}$ (Figura 2B).

$\mathrm{O}$ potássio foi o nutriente acumulado em maior quantidade, com o máximo de 23,6 g planta $^{-1}$, aos 70 DAT, sendo que a maior exigência ocorreu no período de 45 a 60 DAT (Figura 2C). As participações das folhas, caule + ramos e frutos foram, respectivamente, de 17,33 e $50 \%$. A elevada participação dos frutos no conteúdo de $\mathrm{K}$ na planta concorda com outros trabalhos encontrados em literatura que relatam a forte associação entre a maior demanda de $\mathrm{K}$ com a elevada produtividade e qualidade dos frutos de melancia e melão (SUNDSTROM; CARTER, 1983; ZENG; JIANG, 1989; SIMONNE et al., 1992; ZHU et al., 1996 e GRANGEIRO; CECÍLIO FILHO, 2004).

Até 45 DAT, observou-se que a quantidade total acumulada de $\mathrm{K}$ pela planta encontrava-se distribuída entre folhas e caule + ramos. A partir de então, o incremento no acúmulo de $\mathrm{K}$ foi maior no fruto, seguido pelo caule + ramos e folhas, com maior demanda observada no período de 45 a 60 DAT. Neste período, a melancieira apresentou um acúmulo de $700 \mathrm{mg}$ de $\mathrm{K} \mathrm{dia}^{-1}$ (Figura 2C). O acúmulo de $\mathrm{K}$ foi contínuo, porém pequeno, nas folhas até o final do ciclo, constatando-se pequeno decréscimo na quantidade de K no caule + ramos. Nos frutos, o acúmulo de $\mathrm{K}$ permaneceu elevado até a colheita, quando atingiu 11,5 g planta ${ }^{-1}$. O comportamento apresentado pela parte vegetativa da planta (folhas e caule + ramos) foi, provavelmente, ocasionado pela grande demanda por K pelos frutos, fazendo com que ocorresse uma redistribuição do nutriente para estes. Del Rio et al. (1994) verificaram em diversas cultivares de melancieira, reduções nos teores de N, P e K nas folhas com o desenvolvimento dos frutos. Araújo et al. (2001) verificaram redução no acúmulo de $\mathrm{P}, \mathrm{K}, \mathrm{Ca}$ e $\mathrm{Mg}$ na parte vegetativa de abobrinha durante a frutificação.

O cálcio foi o terceiro nutriente mais acumulado pela planta, com máximo de 5,22 $\mathrm{g} \mathrm{planta}^{-1}$. A maior demanda ocorreu no período de 45 a 60 DAT (Figura 2D). Entre os nutrientes, foi o que apresentou maior acúmulo nas folhas, sendo estas responsáveis por $66 \%$, caule + ramos por $23 \%$ e os frutos por apenas $11 \%$. Diferentemente do comportamento observado para o $\mathrm{N}$ e o $\mathrm{P}$, onde houve tendência de estabilização destes nas folhas e caule + ramos, nos últimos 10 dias de ciclo as quantidades acumuladas de cálcio nas folhas, caule + ramos e nos frutos foram crescentes até 70 DAT, atingindo respectivamente, 3,$48 ; 1,21$ e 0,6 g planta $^{-1}$ (Figura 2D). Este padrão de distribuição do Ca em favor das folhas resulta do mesmo ser transportado quase que exclusivamente pelo xilema e praticamente não redistribuído (MALAVOLTAet al., 1997). Outro fator que favorece essa situação é a competição entre K e Ca que se faz, também, dentro da planta (MALAVOLTA et al. 1997). O maior fluxo de potássio para o fruto de melancia concorre para diminuir a presença de cálcio. Comportamento semelhante foi verificado para outras hortaliças, como morango (SOUZA, 1976) e melão (SANCHEZ et al., 1998).

O total acumulado de $\mathrm{Mg}$ na planta foi de 2,67 g planta $^{-1}$, com maior demanda no período de 45 a 60 DAT (Figura 2E), coincidindo com o maior incremento de massa seca na planta. As participações das folhas, caule + ramos e frutos foram, respectivamente, de $45 ; 28$ e $27 \%$. As quantidades de $\mathrm{Mg}$ acumuladas no caule + ramos e frutos, a partir de 60 DAT foram muito semelhantes e, no final do ciclo, ambos segmentos apresentaram $0,74 \mathrm{~g}_{\text {planta }}{ }^{-1}$. A partir dos 45 DAT, o caule + ramos apresentou taxa de acúmulo constante, de aproximadamente $0,3 \mathrm{~g} \mathrm{planta}^{-1}$. Os frutos tiveram maiores demandas no período de 45 a 60 DAT, quando, ao final deste período, o acúmulo de $\mathrm{Mg}$ correspondeu a $59 \%$ do total acumulado no fruto ao final do ciclo.

$\mathrm{O}$ enxofre foi o macronutriente que apresentou acúmulo em menor quantidade, atingindo aos 70 DAT valor estimado de $1,75 \mathrm{~g}_{\text {planta }}{ }^{-1}$. As folhas foram responsáveis por $31 \%$, caule + ramos por $36 \%$ e os frutos por $33 \%$. Nas folhas, o acúmulo foi contínuo ao longo do ciclo, com maior demanda pelo caule + ramos a partir dos 60 DAT (Figura 2F). O acúmulo de enxofre pelos frutos apresentou rápido incremento, acompanhando o acúmulo de massa seca por este segmento da planta, atingindo $0,6 \mathrm{~g}_{\text {planta }}^{-1}$ de $\mathrm{S}$ nos frutos, na colheita.
No momento da colheita, 70 dias após o transplantio, os frutos correspondiam a $39 \%$ do peso seco da planta. Do total dos nutrientes acumulados pelo híbrido de melancia Nova, os frutos participaram com $38 \%$ do $\mathrm{N}$, $45 \%$ do P, $50 \%$ do K, $11 \%$ do Ca, $27 \%$ do $\mathrm{Mg}$ e $33 \%$ do $\mathrm{S}$. As quantidades totais estimadas de $\mathrm{N}, \mathrm{P}, \mathrm{K}, \mathrm{Ca}, \mathrm{Mg}, \mathrm{S}$ exportadas pelos frutos, considerando uma população de 2.333 plantas ha $^{-1}$ do híbrido Nova, foram, respectivamente, de 20,3; 4,0;26,6; 1,4; 1,6; 1,4 $\mathrm{kg} \mathrm{ha}^{-1}$.

Observa-se, portanto, que as quantidades de nutrientes exportadas pelos frutos representam importante componente de perdas de nutrientes do solo, que deverão ser restituídos, enquanto os nutrientes contidos na parte aérea podem ser incorporados ao solo dentro de um programa de reaproveitamento de restos culturais.

\section{AGRADECIMENTOS}

À FAPESP pelo auxílio financeiro concedido, processo $\mathrm{n}^{\circ}$ 2000/01797-0, para a realização deste trabalho e AGROFLORA/SAKATA na pessoa do Eng ${ }^{\circ}$. Agr ${ }^{\circ}$. Dr. Paulo Della Vecchia.

\section{LITERATURA CITADA}

ARAÚJO, W.F.; BOTREL, T.A.; CARMELLO, Q.A.C.; SAMPAIO, R.A.; VASCONCELOS, M.R.B. Marcha de absorção de nutrientes pela cultura da abobrinha conduzida sob fertirrigação. In: FOLEGATTI, M.V.; CASARINI, E.; BLANCO, F.F.; BRASIL, R.P.C.; RESENDE, R.S. (Cood.) Fertirrigação: flores, frutas e hortaliças. Guaíba: Agropecuária, 2001, v.1, p.67-77. BATAGLIA, O.C.; FURLANI, A.M.C.; TEIXEIRA, J.P.F.; FURLANI, P.R.; GALLO, J.R. Métodos de análise química de plantas. Campinas: IAC, $1983.48 \mathrm{p}$.

DEL RIO, A.; LOPEZ-CANTARERO, I.; ROMERO, L.; SANCHEZ, A.; LORENTE, F.A.; VALENZUELA, J.L. Foliar diagnosis: vegetative index for several cultivars of watermelon. Communications in Soil Science and Plant Analysis, v.25, n.9-10, p.1629-1640, 1994.

FAYAD, J.A. Absorção de nutrientes, crescimento e produção do tomateiro cultivado em condições de campo e estufa. 1998. 81 f. Dissertação (Mestrado em Fitotecnia) - Universidade Federal de Viçosa, Viçosa.

GRANGEIRO, L.C.; CECÍLIO FILHO, A.B. Marcha de absorção de nutrientes pela cultura da melancia. In: REUNIÃO BRASILEIRA DE FERTILIDADE DO SOLO E NUTRIÇÃO DE PLANTAS 25., REUNIÃO BRASILEIRA SOBRE MICORRIZAS 9., SIMPÓSIO BRASILEIRO DE MICROBIOLOGIA DO SOLO 7., REUNIÃO BRASILEIRA DE BIOLOGIA DO SOLO 4., 2002, Rio de Janeiro. Anais... 1 CD-ROM. 
GRANGEIRO, L.C.; CECÍLIO FILHO, A.B Acúmulo e exportação de macronutrientes pelo híbrido de melancia Tide. Horticultura Brasileira, Brasília, v.22, n.1, p.93-97, 2004.

LIMA, A.A. Absorção e eficiência de utilização de nutrientes por híbridos de melão (Cucumis melo L.) 2001. 60 f. Dissertação (Mestrado em Solos e Nutrição de plantas) - Universidade Federal do Ceará, Fortaleza.

LOPEZ-CANTARERO, I.; GUZMAN, M.; VALENZUELA, J.L.; DEL RIO, A., ROMERO, L. Variations in nutrient levels in watermelon cultivars irrigated with saline water: total ions. Communications in Soil Science and Plant Analysis, v.23, n.17-20, p.2809-2822, 1992.

MALAVOLTA, E.; VITTI, G.C.; OLIVEIRA, S.A. Avaliação do estado nutricional das plantas: princípios e aplicações. 2 ed. Piracicaba: POTAFOS, 1997. 319 p.

MARR, C.W.; GAST, K.L.B. Reactions by consumers in a 'farmers' market to prices for seedless watermelon and ratings of eating quality. HortTechnology, v.1, p.105-106, 1991.

MARSCHNER, H. Mineral nutrition of higher plants. San Diego: Academic Press, 1995. 889 p NASCIMENTO, V.M.; FERNANDES, F.M.; MORIKAWA, C.K.; LAURA, V.A.; OLIVEIRA, C.A. Produção de matéria seca e absorção de nutrientes pela melancia (Citrullus lanatus (thumb) Masnf.) em um Latossolo da região do cerrado. Científica, Jaboticabal, v.19, n.2, p.8591, 1991.
RAIJ, B.; ANDRADE, J.C.; CANTARELLA, H. QUAGGIO, J.A. Análise química para avaliação da fertilidade de solos tropicais. Campinas: Secretaria de Agricultura e Abastecimento, APTA e IAC, 2001, $284 \mathrm{p}$.

SANCHEZ, L.R.; SIRONI, J.S.; CRESPO, J.A.P.; PELLICER, C.; LOPEZ, M.D.G. Crecimiento y absorcion de nutrientes del melon bajo invernadero. Investigacion Agraria Produccion Proteccion Vegetales, v.13, n.1-2, p.111-120, 1998. SIMONNE, E.H.; MILLS, H.A.; SMITTLE, D.A Ammonium reduces growth fruit yield and fruit quality of watermelon. Journal of Plant Nutrition, v.15, n.12, p.2727-2741, 1992

SOIL SURVEY STAFF. Keys to soil taxonomy. 5 ed. Virginia: SMSS, 1992. 556 p. (Technical monograph, 19)

SOLIS, F.A.M.; HAAG, H.P.; MINAMI, K.; DIEHL. W.J. Nutrição mineral de hortaliças. LVI Acumulação de nutrientes na cultura do pepino (Cucumis sativus L.) var. Aodai cultivado em condições de campo. Anais da Escola Superior de Agricultura Luiz de Queiroz, Piracicaba, v.39, p.697-737, 1988.

SOUZA, A.F. Absorção de nutrientes por quatro cultivares de morangueiro (Fragaria spp). 1976 130 f. Dissertação (Mestrado em Solos e Nutrição de Planta) - Escola Superior "Luiz de Queiroz", São Paulo.
SUNDSTROM, F.J.; CARTER, S.J. Influence of $\mathrm{K}$ and $\mathrm{Ca}$ on quality and yield of watermelon. Journal American Society for Horticultural Science, v.108, n.5, p.879-881, 1983.

TRANI, P.E.; PASSOS, F.A.; NAGAI, H.; MELO, A.M.T. Melão e melancia. In: RAIJ, B.V.; CANTARELLA, H.; QUAGGIO, J.A.; FURLANI, A.M.C. Recomendações de adubação e calagem para o estado de São Paulo. Campinas, $2^{\text {a }}$ edição, 1997, p. 181. (Boletim Técnico, 100)

ZENG, Q.Y.; JIANG, X.L. Influence of potash fertilizers containing chlorine on the quality of watermelon. Soils, v.20, n.3, p.144-146, 1989. ZHU, H.X.; ZHANG, X.; SHEN, A.; SUN, CH. Studies on the nutrient uptake and balance fertilization of watermelon. Acta Horticulturae Sinica, v.23, n.2, p.145-149, 1996. 